\title{
Perforación visceral en liposucción. Medicina basada en evidencia
}

\author{
Visceral perforation in liposuction. Evidence based medicine
}

\author{
Dra. Livia Contreras-Bulnes, ${ }^{*}$ Dr. Jesús Cuenca-Pardo, ${ }^{\ddagger}$ \\ Dr. César Quintana-Vilchis, ${ }^{\S}$ Dra. Estela Vélez-Benítez ${ }^{\|}$
}

Palabras clave: Liposucción abdominal, liposucción, lipoaspiración, complicaciones postoperatorias, perforación intestinal, perforación del intestino delgado, perforación de intestino, perforación de colón, perforación de vísceras, peritonitis,

fascitis necrosante.

Keywords: Abdominal liposuction, liposuction, lipoaspiration, postoperative complications, intestinal perforation, small bowel perforation, bowel perforation, colonic perforation, visceral perforation, peritonitis, necrotizing fasciitis.

* Coordinadora del Comité de Seguridad de la Asociación Mexicana de Cirugía Plástica, Estética y Reconstructiva, A. C. ¥ Asesor del Comité de Seguridad de la Asociación Mexicana de Cirugía Plástica, Estética y Reconstructiva, A. C. Coordinador del Comité de Seguridad de la FILACP.

$\S$ Cirujano General adscrito al Centro Médico «Lic. Adolfo López Mateos», SSA-ISEM.

\section{RESUMEN}

Las perforaciones viscerales son una severa complicación de la liposucción, no se conoce con exactitud su incidencia y factores de riesgo. Realizamos una revisión sistemática con la finalidad de encontrar los factores de riesgo y emitir recomendaciones de seguridad. Las publicaciones que encontramos del tema son escasas y con nivel de evidencia IV y V. Las perforaciones viscerales son la segunda causa de muerte por lipoaspiración. Los factores atribuidos a esta complicación son la falta de experiencia del cirujano y otros relacionados con los pacientes, como la obesidad, abdomen globoso, presencia de hernias, cicatrices y adherencias. Las perforaciones son de dos tipos: 1) De extrema urgencia. Se manifiestan durante la cirugía o en las primeras horas de postoperatorio, en este grupo se incluye el neumotórax a tensión y la hemorragia por lesión de órganos o grandes vasos; estas afecciones requieren de un tratamiento inmediato para evitar la muerte del paciente. 2) Lesiones de los intestinos. Se presentan en una forma insidiosa varios días después de la cirugía, la manifestación cardinal es el dolor atípico que se puede acompañar de obstrucción intestinal, fiebre y deterioro general; el retraso en el tratamiento conlleva una mala evolución y alta mortalidad. La laparotomía exploradora es un procedimiento diagnóstico de salvamento que se debe realizar en urgencia extrema, aun cuando no se cuente con los estudios radiográficos. En la mayoría de los casos el problema mayor no es el incidente de la lesión, sino la falla en reconocerla.

\section{ABSTRACT}

Visceral perforations are a severe complication of liposuction; the incidence and risk factors are not precisely known. We carried out a systematic review in order to find the risk factors and issue safety recommendations. We found few publications on the subject and with IV and $V$ level of evidence. Visceral perforations are the second cause of death from lipoaspiration. The factors attributed to this complication are the surgeon's lack of experience and factors related to patients, such as obesity, globose abdomen, presence of hernias, scars and adhesions. The perforations are of two types: the first group, of extreme urgency, manifest during surgery or in the first postoperative hours. This group includes tension pneumothorax and hemorrhage due to injury to organs or large vessels. These conditions require immediate treatment to avoid the death of the patient. The second group is bowel injuries. They occur in an insidious way, several days after surgery, the cardinal manifestation being atypical pain, which may be accompanied by intestinal obstruction, fever and general deterioration. Delaying the treatment leads to poor evolution and high mortality. The exploratory laparotomy is a lifesaving diagnostic procedure that must be performed urgently, even if radiographic studies are not available. In most cases the main problem is not the incident of the injury, but the failure to recognize it.

\section{INTRODUCCIÓN}

$\mathrm{E}^{\mathrm{n}}$ n el año 2017, la ISAPS registró 1,573,680 cirugías de liposucción en todo el mundo; en México se realizaron 73,231 liposucciones. ${ }^{1}$ Las causas de muerte atribuidas a la liposucción son varias, la perforación visceral es una de las más frecuentes, con una incidencia de 7.81 a $15 \% .^{2-4}$ Se reportan 14 casos de perforación por cada 100,000 liposucciones y varios autores mencionan que existe un subregistro de esta complicación, por lo que no conocemos su verdadera incidencia. ${ }^{5-7}$

Las perforaciones viscerales durante una liposucción se atribuyen a dos causas importantes: la técnica quirúrgica, en especial la experiencia del cirujano y las condiciones estructurales del paciente. Algunos factores de 
" Integrante del Comité de Seguridad de la Asociación Mexicana de Cirugía Plástica, Estética y Reconstructiva, A. C.

Los autores de este artículo no tienen conflicto de intereses que declarar.

Recibido:

24 enero 2019 Aceptado para publicar: 04 marzo 2019 riesgo que relacionan al paciente son la obesidad, abdomen globoso, presencia de hernias umbilicales, eventraciones, cicatrices abdominales y adherencias fibrosas ocasionadas por liposucciones previas. ${ }^{4-7}$

La estructura que se afecta con más frecuencia es el intestino delgado en su porción del íleon, seguida del intestino grueso, hígado, bazo, vena cava, arteria circunfleja profunda, pleura y uréteres. Las perforaciones combinadas se presentan en $10 \%$, colon e íleon en $7.5 \%$ e hígado y pleura en $2.5 \%$. El hallazgo cardinal en los casos de perforación de intestinos es el dolor abdominal persistente atípico y la obstrucción intestinal. La mayoría de las perforaciones no se detectan durante la cirugía y el retraso en el tratamiento se relaciona con la mortalidad (Infografía). ${ }^{4-11}$

Los objetivos de este trabajo son alertar a los cirujanos del riesgo latente que existe en los procedimientos de liposucción de ocasionar una perforación visceral y emitir recomendaciones basadas en la mejor evidencia médica disponible para prevenir, detectar y tratar esta complicación.

\section{METODOLOGÍA}

Realizamos una revisión sistemática en español e inglés en los sitios de información Pub Med, Embase, Cochrane, Medline, Fisterra, Medigraphic y Google académico. Las palabras clave en español que utilizamos fueron: liposucción abdominal, liposucción, lipoaspiración, lipoplastia por succión o lipectomía por succión, escultura corporal, contorno corporal y complicaciones postoperatorias, perforación intestinal, perforación del intestino delgado, perforación de intestino, perforación de colón, perforación de vísceras, peritonitis y fascitis necrosante. Las palabras en inglés fueron: abdominal liposuction, liposuction, suction lipoplasty, suction lipectomy, body sculpture, liposculpture, body contouring and postoperative complications, intestinal perforation, small bowel perforation, bowel perforation, colonic perforation, visceral perforation, peritonitis y necrotizing fasciitis. Con los datos obtenidos elaboramos una infografía como guía rápida y sencilla con la información suficiente que permita al cirujano tomar una decisión en un caso extremo de perforación.

\section{Choque por hemorragia: perforación de bazo, hígado o grandes vasos}

El choque hemorrágico puede producirse por el sangrado local en el tejido subcutáneo, por una aspiración traumática o en pacientes con predisposición al sangrado como resultado de una discrasia sanguínea, por deterioro de la función plaquetaria debido a la ingesta de aspirina, vitamina $E$, vino tinto y algunos suplementos como el ajo. Las otras causas son producidas por la pérdida de sangre en perforaciones de hígado, bazo o grandes vasos. Las manifestaciones del choque en los casos más severos se presentan durante la cirugía o en el postoperatorio inmediato. Algunas laceraciones viscerales pueden provocar una pérdida sanguínea menor y el choque se puede presentar varias horas después de la operación. ${ }^{4-7,9}$

La lesión de la cava es más frecuente que la aorta y puede no estar asociada a perforaciones intestinales. La aspiración con el paciente en decúbito lateral puede hacer que la cánula penetre al retroperitoneo sin afectar los intestinos. La TAC o resonancia magnética con medio de contraste, o angiografía pueden identificar la fuga y el vaso lesionado. En vasos de mediano grosor como las circunflejas, algunos autores utilizan el método de embolizar para controlar la hemorragia. ${ }^{4-7,9-11}$

La perforación del hígado o del bazo se produce al chocar la cánula contra el borde costal y desviar la trayectoria hacia la cavidad abdominal. Los factores involucrados son pacientes con costillas prominentes y flacidez importante de la pared abdominal, introducir las cánulas para aspirar en un plano oblicuo al eje de la piel, así como el tipo de cánula, mientras más delgada mayor factor de flexión, lo que puede cambiar la dirección de la punta. ${ }^{4-7,10,11}$

\section{Recomendaciones: ${ }^{4-7,8,10-14}$}

1. En pacientes con abdomen globoso, pared abdominal con marcada flacidez y costillas prominentes se debe tener cuidado especial al realizar la aspiración en los cuadrantes superiores del abdomen.

2. Tener presente siempre la ubicación de la punta de la cánula. 
3. Tener control constante de la dirección de la cánula y percibir con la mano no dominante del cirujano el sitio donde se encuentra la punta.

4. En caso de encontrar resistencia al momento de introducir la cánula, cambiarla de plano y dirección.

5. Realizar la aspiración en un plano paralelo al eje de la piel.

6. En caso de hipotensión se deberá descartar una lesión de órganos.

7. Sí se puede mantener un equilibrio hemodinámico, realizar TAC o RM con medio de contraste para identificar el sitio de la fuga. Una alternativa de tratamiento es la embolización de los vasos que están sangrando.

8. En casos severos, además de las maniobras de reanimación, se deberá realizar una laparotomía exploradora y el control directo del sangrado. Solicitar apoyo de un cirujano con experiencia.

\section{Neumotórax a tensión, perforación de pleura pulmonar}

La perforación de la pleura es una de las lesiones menos frecuentes, puede estar asociada a lesiones de hígado, bazo e intestinos, con factores de riesgo similares. Es una alteración severa, si no se diagnostica y trata en forma oportuna puede ocasionar la muerte del paciente. La perforación produce un efecto de válvula unidireccional en la pleura y desarrolla un neumotórax a tensión. La lesión se produce al aspirar los cuadrantes superiores del abdomen o la zona inframamaria. Las manifestaciones clínicas son disnea, desaturación de oxígeno y taquicardia, ausencia de ruidos respiratorios, aumento en la resistencia para ventilar al paciente, lateralización de la tráquea al lado opuesto de la lesión pleural, ingurgitación yugular e hipotensión súbitas. La confirmación del diagnóstico se realiza con una radiografía simple de tórax, la cual se puede realizar en el quirófano durante la cirugía o en el área de recuperación. Si no hay la posibilidad de la radiografía, el diagnóstico de neumotórax a tensión tiene que ser clínico y se debe resolver en forma inmediata. El manejo inicial es con una punción en el segundo espacio intercostal a nivel de la línea medio clavicular y el tratamiento definitivo será la colocación de un tubo torácico conectado a un equipo de sello de agua. ${ }^{4-7}$

$$
\text { Recomendaciones: }{ }^{4-7,12-14}
$$

1. Recordar que los pacientes con costillas prominentes, abdomen globoso y flacidez de la pared abdominal tienen riesgo de perforación de hígado, bazo y pleura.

2. Tener cuidado especial al realizar la liposucción en los cuadrantes superiores del abdomen y en la zona costal.

3. Evitar la introducción perpendicular u oblicua de la cánula al plano cutáneo.

4. Si hay resistencia cambiar la cánula de plano y dirección.

5. En todo paciente que tenga desaturación de oxígeno y dificultad para su ventilación durante la cirugía se deberá descartar un neumotórax.

6. Cuando se realice liposucción en los cuadrantes superiores del abdomen y los flancos se deberá proceder a la auscultación de los campos pulmonares antes de terminar el procedimiento. Es recomendable mantener al paciente en observación un mínimo de 24 horas después de la cirugía.

7. Si existe la sospecha o la duda de una perforación, tomar una radiografía de tórax.

8. Cuando no se cuente con el estudio radiográfico en forma inmediata, el diagnóstico se deberá hacer clínicamente.

9. En caso de que se confirme un neumotórax se deberá actuar rápidamente, con punción en el segundo espacio intercostal a nivel de la línea axilar media.

\section{Peritonitis, fascitis necrosante, perforación intestinal}

Las vísceras que con más frecuencia se dañan por una perforación durante la liposucción son las que se encuentran en la zona alrededor del ombligo, como el intestino delgado en sus porciones del íleon y yeyuno. El colon puede ser dañado en todas sus porciones, la más afectada es la transversa. ${ }^{4-7,15-25}$

La zona de mayor riesgo está localizada alrededor del ombligo, algunas características estructurales lo convierten en una zona muy vulnerable. Las hernias umbilicales y el abdo- 
men globoso con su mayor prominencia en el ombligo contribuyen para que una cánula de lipoaspiración se encuentre oblicua al plano de la pared abdominal y de la piel. Las adherencias periumbilicales por ligamentos cutáneos de la zona y por el tejido fibroso cicatricial de cirugías previas, incluyendo lipoaspiraciones, producen un aumento de resistencia a la cánula, con una mayor dificultad para la aspiración y aumento del factor de flexión (bending) de la cánula. Estas distorsiones, rigideces y adherencias pueden desviar la dirección de la punta de la cánula con una mayor posibilidad de perforación visceral alrededor de esta zona. ${ }^{4-7,15-25}$

Diferentes estudios reportan que la perforación intestinal comúnmente no se diagnostica en el postquirúrgico temprano, ${ }^{4-7}$ por las características de muchos pacientes que son jóvenes y con buena reserva física, no siempre están presentes todos los signos típicos de perforación intestinal. ${ }^{9}$ En las lesiones del intestino delgado es de esperar una presentación insidiosa, los signos y síntomas son sutiles y se sobreponen a trastornos como íleo postoperatorio. En orden cronológico, la presentación de los signos clínicos son dolor abdominal persistente que no cede con la analgesia postoperatoria, distensión abdominal, rebote positivo, abdomen rígido y dificultad para canalizar gases, así como signos de inflamación sistémica por sufrimiento de los intestinos como fiebre y taquicardia, taquipnea, deshidratación, deterioro de las condiciones generales y acidosis metabólica. El hallazgo cardinal es el dolor abdominal persistente y no habitual, con evolución estacionaria del paciente en el postoperatorio sin tendencia a la mejoría clínica. 4-7,10,15-25

Debido a que el colon tiene una población bacteriana mayor, la lesión es más peligrosa por la rápida propagación del proceso séptico, tanto al peritoneo como a la fascia de la pared abdominal y tejidos subcutáneos. Se puede encontrar necrosis cutánea y fascitis necrosante importante, sepsis y choque séptico. ${ }^{4-7,15-25}$

Ante la presencia de una evolución no satisfactoria se debe revalorar cuidadosamente al paciente y no apresurar el alta. Se debe establecer un monitoreo estricto, valoración por el cirujano general gastroenterólogo y solicitar algunos exámenes. La biometría hemática será de utilidad para determinar leucocitosis con neutrofilia (datos de sufrimiento de asa), proteína $\mathrm{C}$ reactiva como un marcador inflamatorio confiable de procesos agudos intraperitoneales, telerradiografía de tórax para detección de aire libre subdiafragmático, ultrasonido abdominal para detección de líquido libre intraperitoneal consecutivo a una hemorragia o al acúmulo de líquido secundario al daño intestinal. Tomografía axial computarizada con medio de contraste, esta técnica es la más específica para la detección de perforación intestinal. La mayoría de autores recomienda la laparotomía exploradora como un procedimiento de salvamento que se debe realizar en los casos sospechosos de lesión intestinal, aun cuando no se cuente con los estudios radiográficos. ${ }^{4-9,15-25}$

La diferencia entre un daño limitado o extenso depende de la localización de la lesión y de la rapidez del diagnóstico y tratamiento. Cuando se realiza un diagnóstico temprano, el asa intestinal afectada se puede reparar con cierre directo sin requerir una estoma. En los casos en los que el diagnóstico y tratamiento se realicen después del tercer día, se recomienda realizar una estoma, desbridamiento del tejido necrótico, dejar las heridas abiertas y mantener a los pacientes en una Unidad de Terapia Intensiva para dar soporte de vida. ${ }^{4-9,15-25}$

\section{Recomendaciones: ${ }^{4-7,12-14,26}$}

1. En todos los pacientes a los que se realizará una liposucción del abdomen, se deberán buscar factores de riesgo: obesidad, abdomen globoso, flacidez de la pared abdominal, diastasis de rectos, hernias umbilicales, cicatrices por cirugías, adherencias e irregularidades por liposucciones previas.

2. Es recomendable solicitar un ultrasonido en el preoperatorio para determinar las condiciones de la pared abdominal, el grosor del panículo adiposo, el tamaño de las vísceras abdominales y la grasa intrabdominal.

3. La infiltración de la solución tumescente se debe realizar con cánulas mayores de $4 \mathrm{~mm}$ de diámetro. No usar cánulas delgadas.

4. La introducción de las cánulas se deberá hacer en un plano paralelo al eje de la piel y de la pared abdominal. Evitar la introducción con las cánulas oblicuas. 
5. Tener cuidado especial al realizar la aspiración alrededor del ombligo, si hay resistencia por adherencias cicatriciales, cambiar la dirección y el plano. En presencia de hernia umbilical es preferible la succión en forma centrífuga a la cicatriz umbilical. ${ }^{15}$

6. Todos los pacientes a los que se les realice liposucción del abdomen deberán permanecer en observación hospitalaria mínimo 24 horas y revisión en consultorio a las 48 horas.

7. Paciente con persistencia de dolor abdominal no habitual, descartar una perforación intestinal.

8. Pacientes con distensión abdominal, dificultad para canalizar gases, abdomen rígido doloroso, fiebre y taquicardia se deberán internar para su estudio. Solicitar exámenes de laboratorio, placas simples de abdomen y tórax, ultrasonido y TAC o RM con medio de contraste.

9. En los casos de sospecha de lesión intestinal, solicitar la valoración por un cirujano general con experiencia en cirugía abdominal preoperatoria.

10. En los casos de sospecha de lesión intestinal, solicitar la valoración por un cirujano general gastroenterólogo.

11. Cuando exista una fuerte sospecha de perforación intestinal, aun cuando no se cuente con TAC o RM, realizar una laparotomía exploradora.

12. El diagnóstico y tratamiento de las perforaciones debe ser oportuno, el retraso hará más complejo el tratamiento, incrementará la estancia hospitalaria y tendrá mayor riesgo de muerte.

\section{DISCUSIÓN}

Realizamos una revisión sistemática tratando de encontrar la mejor evidencia médica disponible publicada. Los niveles de evidencia de las publicaciones encontradas fueron IV y V, la mayoría corresponden al reporte de un caso o a una serie de casos. Otras publicaciones que utilizamos para este trabajo fueron algunas revisiones e ideas e innovaciones. Hacen falta estudios clínicos controlados, meta-análisis, investigación en laboratorio y en cadáveres para poder emitir recomendaciones con un mayor fundamento.
Hay reportados 14 casos de perforación por cada 100,000 liposucciones; es la segunda o tercera causa reportada de muertes en lipoaspiración. ${ }^{2-5}$ Varios autores mencionan que existe un subregistro de esta complicación, por lo que no conocemos su verdadera incidencia. Las complicaciones y las muertes deben reportarse, incluso aquéllas que ocurren dentro de los 30 días posteriores a la liposucción. ${ }^{5-7,27}$ En el Comité de Seguridad llevamos un registro de los casos que hemos detectado y señalamos los factores de riesgo. La Secretaría de Salud en México, a través de la COFEPRIS, exige el registro de eventos adversos y el reporte puede ser anónimo y confidencial. ${ }^{28}$ Es necesario fomentar la cultura del reporte de las complicaciones. Al contar con un mejor registro podremos determinar los factores de riesgo y establecer estrategias para disminuir o abatir esta complicación.

Varios autores consideran que el principal factor de riesgo de las perforaciones viscerales por lipoaspiración es la falta de experiencia del cirujano. ${ }^{4-7,12-14,26}$ Aunque resulta lógica y plausible esta premisa, en las publicaciones revisadas no encontramos datos suficientes para confirmar esta aseveración. En estudios prospectivos que realizaremos incluiremos este factor para su estudio. Hemos de fomentar el adiestramiento personalizado de los cirujanos jóvenes para evitar y prevenir las perforaciones.

Lehnhardt encontró que la falta de restricciones legales incrementa la ejecución de liposucción por personal no calificado. Muchos de estos procedimientos son realizados por cirujanos no plásticos y por personal no médico; además, es frecuente que estos procedimientos se efectúen como cirugía ambulatoria en oficinas o consultorios. Esto ocasiona un incremento en el número de complicaciones relacionadas a la liposucción. Una de las complicaciones más severas fue la perforación visceral hecha por las cánulas de aspiración, con una alta mortalidad. ${ }^{4}$ La liposucción es un procedimiento de alto riesgo y se requiere de un adiestramiento personalizado de los cirujanos. No se podrá efectuar hasta el dominio pleno de las capacidades que la hagan un procedimiento seguro.

En los casos reportados existen alteraciones estructurales comunes relacionadas a las perforaciones viscerales por lipoaspiración, 
las más frecuentes son la obesidad, abdomen globoso, presencia de hernias umbilicales, eventraciones, cicatrices abdominales y adherencias fibrosas ocasionadas por liposucciones previas. ${ }^{4-7,15-25}$ La mejor estrategia para evitar esta complicación es la detección preoperatoria de estas alteraciones, con una exploración dirigida y apoyada con un estudio de ultrasonido. El ultrasonido ayudará a identificar defectos de la pared abdominal, el tamaño y dilatación de las vísceras abdominales, el grosor y densidad del panículo adiposo y la presencia de adherencias cicatriciales y hernias.

Durante una liposucción del abdomen, el choque hemorrágico se puede presentar por lesión de grandes vasos o por perforación del hígado o bazo; ${ }^{4-7,9-11,12}$ es una complicación que se tiene que resolver en forma inmediata cuando existe la sospecha; incluso, cuando no se cuente con una TAC o RM, se deberá realizar una exploración quirúrgica que incluya el retroperitoneo, las ingles y los sitios donde emergen los grandes vasos. La cava puede perforarse, aun sin lesión de intestinos, lo que implica que la cánula de aspiración ingresó al retroperitoneo sin atravesar la cavidad abdominal, con un acceso lateral.

La perforación de hígado o bazo puede ser única o estar asociada a perforaciones de otras estructuras como el colon o la pleura. Se produce al chocar la cánula contra el borde costal y desviar la trayectoria hacia la cavidad. 4-7,9,11,12 En los pacientes obesos con abdomen globoso y flácido y costillas prominentes se deberá tener especial cuidado cuando se aspiren los cuadrantes superiores del abdomen o los flancos.

La perforación de la pleura es una alteración severa; el efecto de válvula unidireccional en la pleura puede producir un neumotórax a tensión. Cuando se realiza una aspiración en los cuadrantes superiores del abdomen o la zona inframamaria y se presenta disnea en forma súbita, desaturación de oxígeno, taquicardia y aumento en la resistencia para ventilar al paciente, debemos sospechar una perforación de pleura con formación de neumotórax. ${ }^{4-7}$ Una radiografía de tórax confirmará el diagnóstico; sin embargo, ante la sospecha clínica, se deberá actuar rápidamente. Cuando no se cuente con radiografía se deberá realizar una punción en el segundo espacio intercostal; el tratamiento definitivo será la colocación de un tubo torácico conectado a un equipo de sello de agua. El retraso en el tratamiento puede ser mortal para el paciente.

Las vísceras que con más frecuencia se dañan por una perforación son las que se encuentran en la zona alrededor del ombligo; el íleon y yeyuno se afectan hasta en $62 \%$ de los casos (Infografía). ${ }^{4-7,15-25}$ Esta zona es muy vulnerable por sus características estructurales: abdomen globoso con su mayor prominencia en el ombligo, gran grosor del panículo adiposo, presencia habitual de hernias umbilicales, adherencias peri-umbilicales por ligamentos cutáneos de la zona y por el tejido fibroso cicatricial de cirugías previas, incluyendo lipoaspiraciones. Estas características dificultan la introducción de las cánulas y aumentan su factor de flexión, con la posibilidad de desviar inadvertidamente la punta y perforar la pared abdominal.

Las perforaciones del intestino delgado son de una presentación insidiosa; los signos y síntomas son sutiles y se sobreponen a trastornos como íleo postoperatorio. Las lesiones del colon son más peligrosas y propensas a la sepsis con fascitis necrosante y choque séptico. ${ }^{4-7,15-25}$ Las manifestaciones clínicas van del dolor persistente, distensión y rigidez abdominal, dificultad para canalizar gases, fiebre, taquicardia, taquipnea, deshidratación y deterioro de sus condiciones generales a la acidosis metabólica. En varios casos la única manifestación que tuvieron los pacientes fue el dolor y el deterioro general sin tendencia a la mejoría clínica. ${ }^{4-7,10,15-25}$ Los pacientes a los que se les realiza una lipoaspiración deben tener una vigilancia estrecha los primeros siete días. Cuando exista una evolución tórpida sin tendencia a la mejoría se deberá internar al paciente para descartar una perforación visceral.

La laparotomía exploradora es un procedimiento diagnóstico y de salvamento en los casos de perforación visceral, y en ocasiones se debe realizar en extrema urgencia, incluso sin contar con los estudios radiográficos. ${ }^{4-9,15-25} \mathrm{El}$ diagnóstico y tratamiento oportuno realizado por un cirujano general con experiencia en este tipo de eventualidades resolverá satisfactoriamente la mayoría de los casos. El retraso en la atención puede tener consecuencias fatales. En la mayoría de los casos el problema mayor no es el incidente de la lesión, sino la falla a reconocerla. 


\section{CONCLUSIONES}

Las perforaciones viscerales son la segunda causa de muerte por lipoaspiración. Los factores atribuidos a esta complicación son la falta de experiencia del cirujano y los relacionados con los pacientes como la obesidad, abdomen globoso, presencia de hernias umbilicales, cicatrices abdominales y adherencias fibrosas ocasionadas por cirugías y liposucciones previas. Las perforaciones viscerales por liposucción pueden ser de dos tipos: el primer grupo, de extrema urgencia, se manifiesta durante la cirugía o en las primeras horas de postoperatorio; en este grupo se incluye el neumotórax a tensión y la hemorragia por lesión de órganos o grandes vasos. Estas afecciones requieren un tratamiento inmediato para evitar la muerte del paciente. El segundo grupo son las lesiones de los intestinos, las cuales se presentan en forma insidiosa varios días después de la cirugía; la manifestación cardinal es el dolor atípico que se puede acompañar de obstrucción intestinal, fiebre y deterioro general. El retraso en el diagnóstico y tratamiento conlleva una mala evolución y alta mortalidad. La laparotomía exploradora es un procedimiento diagnóstico y de salvamento, que se debe realizar de extrema urgencia, aun cuando no se cuente con los estudios radiográficos. En la mayoría de los casos el problema mayor no es el incidente de la lesión, sino la falla en reconocerla. Todo paciente a quien se le realiza una liposucción del abdomen, debe mantenerse en observación los primeros siete días posteriores a la cirugía. En caso de dolor y evolución tórpida se debe descartar una perforación visceral. Realizamos una revisión sistemática tratando de encontrar la mejor evidencia médica disponible publicada. Los niveles de evidencia de las publicaciones encontradas fueron IV y $\mathrm{V}$, y la mayoría corresponde al reporte de un caso o a una serie de casos. Otras publicaciones que utilizamos para este trabajo fueron algunas revisiones e ideas e innovaciones. Hacen falta estudios clínicos controlados, meta-análisis, investigación en laboratorio y en cadáveres para poder emitir recomendaciones con un mayor fundamento.

\section{REFERENCIAS}

1. ISAPS Global Statistics 2017. Available in: https://www. isaps.org/medical-professionals/isaps-global-statistics/.
2. Grazer FM, Jong RH. Fatal outcomes from liposuction. Census survey of cosmetic surgeons. Plast Reconstr Surg 2000; 105: 436-446.

3. Cuenca-Pardo J, Contreras-Bulnes L, Iribarren-Moreno R, Hernández-Valverde C. Muerte súbita en pacientes de lipoaspiración; recomendaciones preventivas. Cir Plast 2014; 24 (1): 16-30.

4. Lehnhardt M, Homann HH, Daigeler A, Hauser J, Palka P, Steinau HU. Major and lethal complications of liposuction: a review of 72 cases in Germany between 1998 and 2002. Plast Reconstr Surg 2008; 121 (6): 396-403.

5. Bellini E, Grieco MP, Raposio E. A journey through liposuction and liposculpture: review. Ann Med Surg 2017; 24: 53-60.

6. Zakine G, Baruch J, Dardour JC, Flageul G. Perforation of viscera, a dramatic complication of liposuction: a review of 19 cases evaluated by experts in France between 2000 and 2012. Plast Reconstr Surg 2015; 135 (3): 743-750.

7. Chia CT, Neinstein RM, Theodoru SJ. Evidence-based medicine: liposuction. Plast Reconstr Surg 2017; 139: 267-274.

8. Kattapuram TM, Avery LL. Ureteral tear at the ureteropelvic junction: a complication of liposuction. Emerg Radiol 2010; 17 (1): 79-82.

9. Toledo LS, Mauad R. Complications on body sculpture: prevention and treatment. Clin Plast Surg 2006; 33: 1-11.

10. Gialamas E, Oldani G, Modarressi A, Morel Ph, Toso Ch. Liver trauma during combined liposuction and abdominoplasty: a rare but potentially lethal complication. Aesth Surg J 2015; 35 (7): 211-215.

11. Hernett $P$, Koak $Y$, Baker D. Splenic trauma during abdominal wall liposuction: a case report. J R Soc Med 2008; 101: 201-203.

12. Choi H, Shin T. Rupture of a deep circumflex iliac artery after abdominal liposuction: treatment with selective arterial transcatheter embolization. Cardiovasc Intervent Radiol 2009; 32 (6): 12881290.

13. Cardenas-Camarena L, Lozano-Peña A G, Durán $H$, Bayter-Marin JE. Strategies for reducing fatal complications in liposuction. Plast Reconstr Surg Glob Open 2017; 6: 1-5.

14. Dixit VV, Wagh MS. Unfavorable outcomes of Liposuction and their management. Indian J Plast Surg 2013; 46 (2): 377-392.

15. Sharma D, Dalencourt G, Bitterly T, Benotti PN. Small intestinal perforation and necrotizing fasciitis after abdominal liposuction. Aesthet Plast Surg 2006; 30 (6): $712-716$

16. Mallappa $M$, Rangaswamy $M$, Badiuddin MF. Small intestinal perforation and peritonitis after liposuction. Aesthetic Plast Surg 2007; 31: 589-592.

17. Di Candia M, Malata CM. Aesthetic and functional abdominal wall reconstruction after multiple bowel perforations secondary to liposuction. Aesthet Plast Surg 2011; 35 (2): 274-277.

18. Coronado-Malagón M, Tauffer-Carrion LT. Jejunal perforation after abdominal liposuction, bilateral breast augmentation and facial fat grafting. Can J Plast Surg 2012; 20 (3): 197-198. 
19. Raman SR, Pokala N, Cosgrove J, Jamil Z. Colocutaneous fistula after suction lipoplasty: case report and literature review. Ann Plast Surg 2010; 64 (4): 503-505.

20. Reddy AK. Bowel injury following liposuction. A rare complication of cosmetic surgery. Inter Arch Med 2016; 9 (54): 1-3. Available at: www.intarchmed.com and www.medbrary.com.

21. Delliere V, Bertheuil N, Harnois $Y$, Thienot $S$, Gerard $M$, Robert $M$ et al. Multiple bowel perforation and necrotizing fasciitis secondary abdominal liposuction in a patient with bilateral lumbar hernia. Indian J Plast Surg 2014; 47(3): 436-440.

22. Talmor M, Hoffman LA, Lieberman M. Intestinal perforation after suction lipoplasty: a case report and Review of the literature. Ann Plast Surg 1997; 38 (2): 169-172.

23. Ovrebo KK, Grong K, Vindenes H. Small intestinal perforation and peritonitis after abdominal suction lipoplasty. Ann Plast Surg 1997; 38: 642-644.

24. Márquez-Álvarez L, Rodríguez-García R, PalomoAntequera C, Escudero Augusto D, González Pinto I. Perforación intestinal tras liposucción. Cirugía Española 2019; 97 (9): 536-538. https://doi.org/10.1016/j. ciresp.2018.12.006.
25. Fouad M, Mossalam A, Al-Naqeeb N. Samall bowel perforation as a complication of liposuction. $K$ Med J 2005; 37: 54-55.

26. Ezzeddine H, Husari A, Nassar H, Kanso M, Nounou GE, Khalife M, Faraj W. Life threatening complications postliposuction. Aesthet Plast Surg 2018; 42 (2): 384-387.

27. Klein JA. Problems in reporting liposuction deaths. In: Klein JA. Tumescent technique in liposuction. Textbook, Ed Liposuction 2015, 101; 5 (https:// liposuction101.com >liposuction textbook).

28. Dirección General de Calidad y Educación en Salud. Sistema de Registro de Eventos Adversos. desdegces. salud.gob.mx/srea/.

Correspondencia:

\section{Dra. Livia Contreras Bulnes}

Asociación Mexicana de Cirugía Plástica, Estética y Reconstructiva, A.C.

Flamencos Núm. 74,

Col. San José Insurgentes, 03900,

Alcaldía Benito Juárez,

Ciudad de México, México.

E-mail: liviacbulnes@gmail.com 


\section{RECOMENDACIONES DE SEGURIDAD}

\section{Perforación visceral por liposucción}

\section{Diseñador: Jonathan Morales}

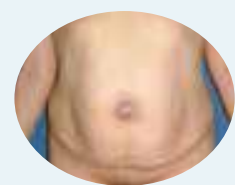

Hernias, diastasis de rectos, debilidad de la pared muscular

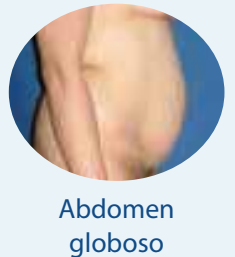

Factores de riesgo de perforaciones viscerales en liposucción

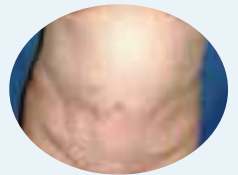

Adherencias y cicatrices posteriores a liposucción

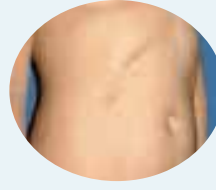

Cicatrices por cirugía abdominal

Las muertes registradas están relacionadas a la edad de los pacientes $y$ al retraso en el tratamiento No se ha encontrado una correlación entre la incidencia y la experiencia del cirujano

Estudios de imagen para detectar defectos de la pared abdominal

- Ultrasonido

- TAC

- Resonancia magnética

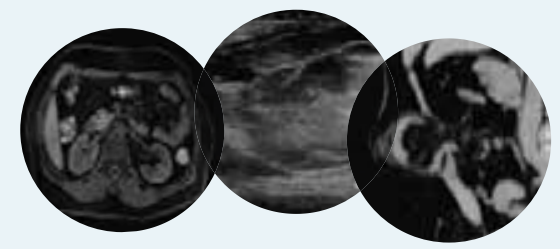

Una maniobra de Valsalva durante el estudio incrementa la sensibilidad

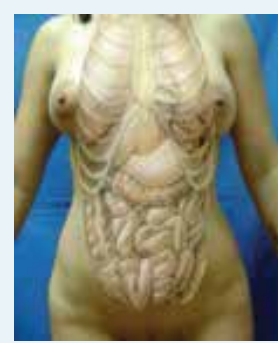

Zona I. Intestino delgado $=62.5 \%$

Zona II. Intestino grueso $=17.5 \%$

Zona III. Órganos bazo $=7.5 \%$

Hígado $=7.5 \%$

Zona IV = Vasos

Sanguíneos cava $=5 \%$ Circunfleja profunda $=2.5 \%$

Zona V = Tórax $2.5 \%$

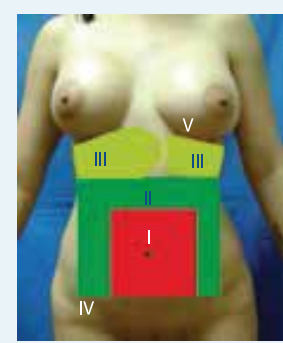

Vísceras dañadas por liposucción Hallazgos de un meta-análisis
Las lesiones combinadas se presentaron en $10 \%$ Colon + ilium en $7.5 \%$; hígado + pleura $=2.5 \%$

Un caso fue por liposucción asistida con mecanismo propulsor; el resto con técnica habitual
La combinación de liposucción con abdomioplastia aumenta el riesgo de perforaciones viscerales

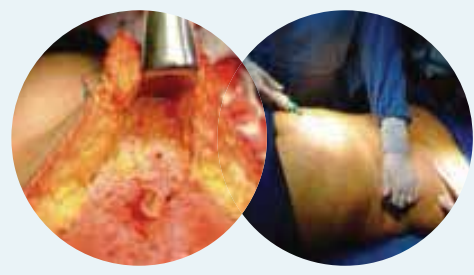

Recomendable antes de la cirugía

\section{La perforación de vísceras es la segunda causa de muerte por lipoaspiración. Es una complicación subestimada}

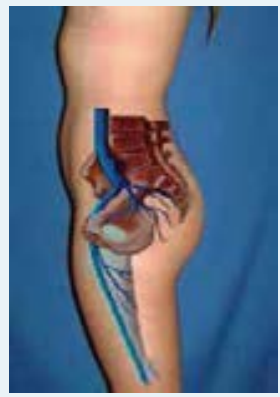

\section{La vena cava fue dañada en $5 \%$ de los casos}

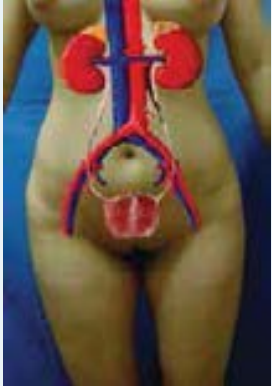

El choque hipovolémico durante la cirugía o en las primeras 6 horas se debe por lesión de:

- Hígado

- Bazo

- Grandes vasos sanguíneos

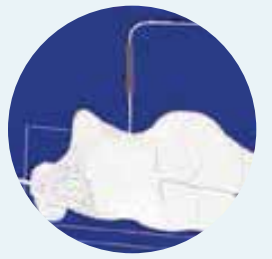

caución

La liposucción de la superficie anterior del abdomen o de la región lumbar, con el paciente en decúbito lateral, conlleva el riesgo de perforación de la cava; el riesgo aumenta si el tronco se encuentra en torsión

- Capacitación del cirujano con expertos

- Buscar factores de riesgos

- Ultrasonido de rutina en todos los pacientes

- Conocer las complicaciones reportadas

- Conocer las recomendaciones de seguridad para prevenir esta complicación 


\author{
Recomendable durante la cirugía \\ Cuidados durante la infiltración y aspiración \\ para prevenir una perforación
}

$\begin{array}{cc}\text { Evitar cánulas } & \text { La infiltración y } \\ \text { delgadas } & \text { aspiración sólo } \\ \text { (infiltración) } & \text { por el cirujano }\end{array}$

\begin{abstract}
Hiperextensión del
abdomen, almohada

en la espalda o

doblar la mesa
\end{abstract}

Conciencia constante de la posición de la cánula

Extremar los cuidados alrededor del ombligo

Especial cuidado en los cambios de región anatómica
En caso de encontrar resistencia, ajustar la dirección de la posición de la cánula

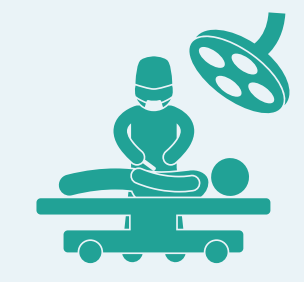

Sentir en todo momento la punta de la cánula

Dirigir la cánula en dirección tangencial

Infiltración cuidadosa de la solución vaso-constrictora

\title{
Recomendable después de la cirugía
}

\section{Mantener en \\ observación \\ mínimo 24 horas \\ Revisar en la \\ consulta a \\ las 48 horas}

\begin{abstract}
Buscar dolor
no habitual y

persistente
\end{abstract}

Buscar datos de obstrucción intestinal

Buscar datos de peritonitis

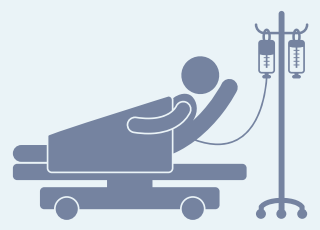

Identificar manifestaciones de sepsis como taquicardia, fiebre
Identificar datos de deshidratación o ataque al estado general

Identificar datos de choque

hipovolémico

Identificar alteraciones cutáneas de las áreas operadas

En caso de duda: hospitalizar a

la paciente y realizar estudios

\section{Todos los pacientes vigilancia: hospital por $\mathbf{2 4}$ horas, consulta por $\mathbf{7}$ días}

\section{Paciente con choque hipovolémico \\ Se debe descartar una perforación de hígado, \\ bazo o vena cava}

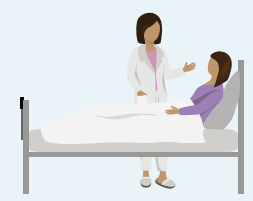

- Mantener en observación

- Solicitar estudios

- Interconsulta con cirujano general

- Laparatomía exploradora

\section{Estudios que se deben realizar a una paciente con sospecha de perforación visceral}

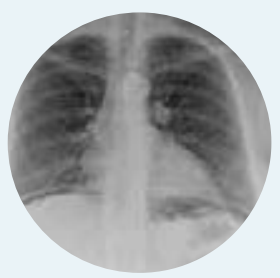

- Placa simple de abdomen. Líquido o aire en cavidades

- Ultrasonido. Líquido o aire libre en cavidades o hematomas alrededor de los órganos dañados

- TAC o resonancia magnética contrastada. Hematomas y fuga del medio de contraste

- Laparatomía exploradora es una medida de salvamento y se deberá utilizar aun cuando no se cuente con los estudios anteriores

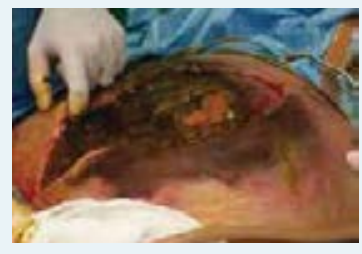

Perforación intestinal por liposucción
El retraso en la atención de los pacientes produce: - Peritonitis

- Necrosis cutánea

- Dermo-fascitis

- Choque séptico

- Muerte
Paciente con dolor náuseas, vómito

alteración del

Se debe descartar una perforación de intestinos 